\title{
Tradução, adaptação cultural e validação do questionário de avaliação sintomática do lipedema (QuASiL)
}

\author{
Translation, cultural adaptation, and validation of a lipedema symptoms questionnaire \\ Alexandre Campos Moraes Amato ${ }^{1,2}$ (D), Fernando Campos Moraes Amato², Daniel Augusto Benitti³, \\ Ricardo Virgínio dos Santos ${ }^{1,2}$
}

\begin{abstract}
Resumo
Contexto: O lipedema é caracterizado como uma deposição anormal de gordura em glúteos e pernas bilateralmente, que pode ser acompanhada por edema ortostático em mulheres. Foi publicado questionário de avaliação sintomática em lipedema em alemão e inglês para avaliação de evolução de tratamento. Objetivos: Tradução, adaptação cultural e validação do questionário de avaliação sintomática de lipedema para português brasileiro. Métodos: $\mathrm{O}$ processo consistiu em três traduções e duas retrotraduções realizadas por tradutores independentes, seguida da elaboração de versão consensual e adaptação conforme compreensão das pacientes. O questionário foi convertido em versão digital, aplicado em 56 voluntárias e em 154 pacientes de consultório vascular e correlacionado com volumetria por bioimpedância segmentar. Resultados: As 20 pacientes do pré-teste eram do sexo feminino e as questões foram compreendidas por pelo menos $90 \%$ das entrevistadas na fase final. Voluntárias mostraram compreensão de $96,4 \%$, com tempo médio de resposta de 4 minutos. Em 154 pacientes, o volume dos membros mostrou correlação positiva com a intensidade dos sintomas avaliada pelo questionário traduzido e correlação fraca e moderada em apenas 3 perguntas, num total de 15 com nível de escolaridade. Conclusões: A versão traduzida e adaptada culturalmente para o português do Brasil do questionário de avaliação sintomática do lipedema (QuASiL) é um instrumento prático, de fácil e rápida aplicação, que pode ser utilizado em nossa população. Ainda são necessários estudos adicionais para avaliar a sensibilidade do instrumento para o auxílio no diagnóstico do lipedema.
\end{abstract}

Palavras-chave: questionários; tradução; comparação transcultural; lipedema; obesidade; linfedema.

\begin{abstract}
Background: Lipedema is characterized as abnormal bilateral deposition of fat in the buttocks and legs that may be accompanied by orthostatic edema in women. A questionnaire for assessment of lipedema symptoms has previously been published in German and English to assess treatment progress. Objectives: To translate, culturally adapt, and validate the lipedema symptoms assessment questionnaire for Brazilian Portuguese. Methods: The process involved three translations and two back-translations performed by independent translators, followed by construction of a consensus version and adaptation according patients' comprehension. The questionnaire was converted into a digital version and administered to 56 volunteers and then administered to 154 patients from a vascular clinic and correlated with volume determined by segmental bioimpedance. Results: The 20 pre-test patients were female and at least $90 \%$ of the interviewees were able to understand the questions in the final phase. Volunteers had a $96.4 \%$ rate of comprehension of the digital online version and a mean completion time of 4 minutes. In 154 patients, limb volume was positively correlated with intensity of symptoms, as assessed by the translated questionnaire, and 3 out of 15 questions were weakly correlated with educational level. Conclusions: The translated and culturally adapted Brazilian Portuguese version of the lipedema symptoms assessment questionnaire (QuASiL) is a practical tool that is easy and quick to administer and can be used in our population. Additional studies are still needed to assess the instrument's sensitivity as an aid for diagnosis of lipedema.
\end{abstract}

Keywords: questionnaires; translation; cross-cultural comparison; lipedema; obesity; lymphedema.

Como citar: Amato ACM, Amato FCM, Benitti DA, Santos RV. Tradução, adaptação cultural e validação do questionário de avaliação sintomática do lipedema (QuASiL). J Vasc Bras. 2020;19:e20200049. https://doi.org/10.1590/1677-5449.200049

${ }^{1}$ Universidade de Santo Amaro - UNISA, Disciplina de Cirurgia Vascular, São Paulo, SP, Brasil.

${ }^{2}$ Amato - Instituto de Medicina Avançada, São Paulo, SP, Brasil.

${ }^{3}$ Medical Valens Center, Campinas, SP, Brasil.

Fonte de financiamento: Nenhuma.

Conflito de interesse: Os autores declararam não haver conflitos de interesse que precisam ser informados.

Submetido em: Abril 16, 2020. Aceito em: Maio 19, 2020. 


\section{INTRODUÇÃO}

O lipedema foi descrito pela primeira vez em 1940 pelos doutores Edgar Van Nuys Allen e Edgar Alphonso Hines Jr. na Mayo Clinic e foi caracterizado como uma deposição anormal de gordura em glúteos e pernas bilateralmente, que pode ser acompanhada por edema ortostático ${ }^{1,2} \mathrm{em}$ mulheres. A fisiopatologia e a epidemiologia do lipedema é até hoje pouco compreendida, embora sejam sugeridos seu aspecto genético e sua influência hormonal em sintomas inflamatórios cíclicos ${ }^{3}$. Sendo assim, apesar de condição distinta, é frequentemente confundido com doenças mais frequentemente diagnosticadas, como a obesidade e o linfedema ${ }^{4,5}$. O diagnóstico é clínico e é tipicamente definido pela desproporção simétrica do acúmulo de gordura nas extremidades inferiores com queixas de edema ortostático ${ }^{4}$, frequentemente acompanhada por dor em peso, cansaço, tensão ou de difícil caracterização; constante ou ao toque nas áreas mais afetadas; com intensidade variável e sem irradiação. Os pés são poupados desse aumento de tamanho, exceto no estágio avançado de lipolinfedema, no qual o edema do pé ocorre secundariamente à insuficiência linfática inexistente em estágios anteriores $^{6,7}$. O edema que poupa os pés é sinal importante para distinguir o lipedema da obesidade comum. A parte de cima do corpo (tronco) é poupada até os estágios mais avançados da doença, embora existam alguns subtipos de lipedema atípicos que podem variar do padrão esperado de acúmulo de gordura em extremidade inferior ${ }^{8,9}$. As áreas afetadas pelo lipedema frequentemente apresentam hematomas, dor e aumento de sensibilidade, acompanhados por queixas sistêmicas de fadiga e diminuição de condição física e força muscular. O início dos sintomas frequentemente começa durante a puberdade ou a jovem adulta, embora algumas pacientes possam iniciar até mais tardiamente ${ }^{4}$. Medidas conservadoras das estimativas da prevalência de lipedema na população em geral variam de 0,06 a $10 \%{ }^{4}$.

Um questionário específico para o lipedema, sem nome, foi elaborado na Alemanha para avaliação sintomática pré e pós-operatória de lipedema e publicado por Rapprich et al. ${ }^{10} \mathrm{em}$ alemão e inglês ${ }^{11}$. O questionário foi baseado no questionário de qualidade de vida para pacientes com doenças linfáticas ${ }^{12} \mathrm{e}$ adaptado para incluir quinze critérios autoavaliados por escala analógica de 0 a 10 . O questionário, baseado em avaliação de qualidade de vida, pode ser considerado uma escala sintomática do lipedema e não foi validado para uso como critério diagnóstico.

O questionário original é interpretado como intensidade dos sintomas por meio de uma pontuação derivada de avaliação analógica e visual, que pode variar de 0 a 150 , sendo que 0 representa nenhuma queixa sintomática e 150 , sintomas com o maior impacto em qualidade de vida. Tendo em vista a rara disponibilidade de instrumentos objetivos para avaliação de lipedema no Brasil e no mundo e que dados referentes à qualidade de vida são importantes para a seleção e interpretação da evolução clínica, justifica-se este estudo tendo como objetivo traduzir para a língua portuguesa e adaptar culturalmente para a população brasileira o questionário, além de validá-lo na prática clínica.

\section{MÉTODO}

O presente estudo segue as normas do Conselho Nacional de Saúde, referentes à resolução n 466/12 sobre pesquisa envolvendo seres humanos. Também segue a declaração de Helsinki e foi aprovado pelo Comitê de Ética em Pesquisa da Plataforma Brasil sob protocolo CAAE: 09590919.6.0000.0081.

\section{Método de tradução e adaptação cultural}

$\mathrm{O}$ processo de equivalência linguística iniciou-se por meio de contato com o autor original para definição de conceitos e autorização para utilização destes. A tradução e a adaptação cultural do instrumento foram feitas com base em diretrizes existentes ${ }^{13,14}$, consistindo em tradução, retrotradução, revisão por painel de especialistas e adaptação cultural (Figura 1).

Os itens da versão original do questionário em inglês foram traduzidos inicialmente por um profissional de saúde, independente, brasileiro, com grande conhecimento da língua inglesa e que estava ciente dos objetivos da pesquisa. O questionário em alemão foi traduzido separadamente por dois tradutores com grande conhecimento da língua alemã, cientes dos objetivos da pesquisa. Optou-se por duas versões do original em alemão devido ao autor ser nativo alemão. Foi enfatizada a importância da tradução conceitual em detrimento de uma tradução literal. Foram, assim, obtidas as versões de número 1 a 3 em português brasileiro. As traduções 1 a 3 foram avaliadas e unidas em versão consensual por dois médicos cientes dos objetivos e especialistas em lipedema. A versão consensual foi vertida para o alemão por dois professores de alemão, independentes, nativos, que não participaram da etapa anterior e não tinham conhecimento dos objetivos nem dos conceitos apresentados no questionário. Em seguida, foi feita a comparação do instrumento original com as duas novas versões em alemão. Um painel de especialistas composto por dois médicos documentou e analisou as discrepâncias encontradas. Alguns tempos verbais e sentenças em português foram reescritos até se obter um consenso, gerando, assim, a versão 4 em português. 
A versão 4 em português do questionário foi aplicada a um grupo de 10 indivíduos para avaliar o grau de compreensão das questões, sendo selecionadas aquelas que não apresentavam boa compreensão. O painel de especialistas avaliou esses itens e os adaptou com o mesmo conceito, porém de mais fácil compreensão, utilizando, inclusive, sugestões dos próprios respondentes, procurando não alterar a estrutura e as propriedades de avaliação dessas questões e gerando a versão 5 do questionário. Essa versão 5 (Tabela 1) foi aplicada a outro grupo de 10 indivíduos, selecionados aleatoriamente no mesmo

Avaliação consensual Versão consensual Retrotradução

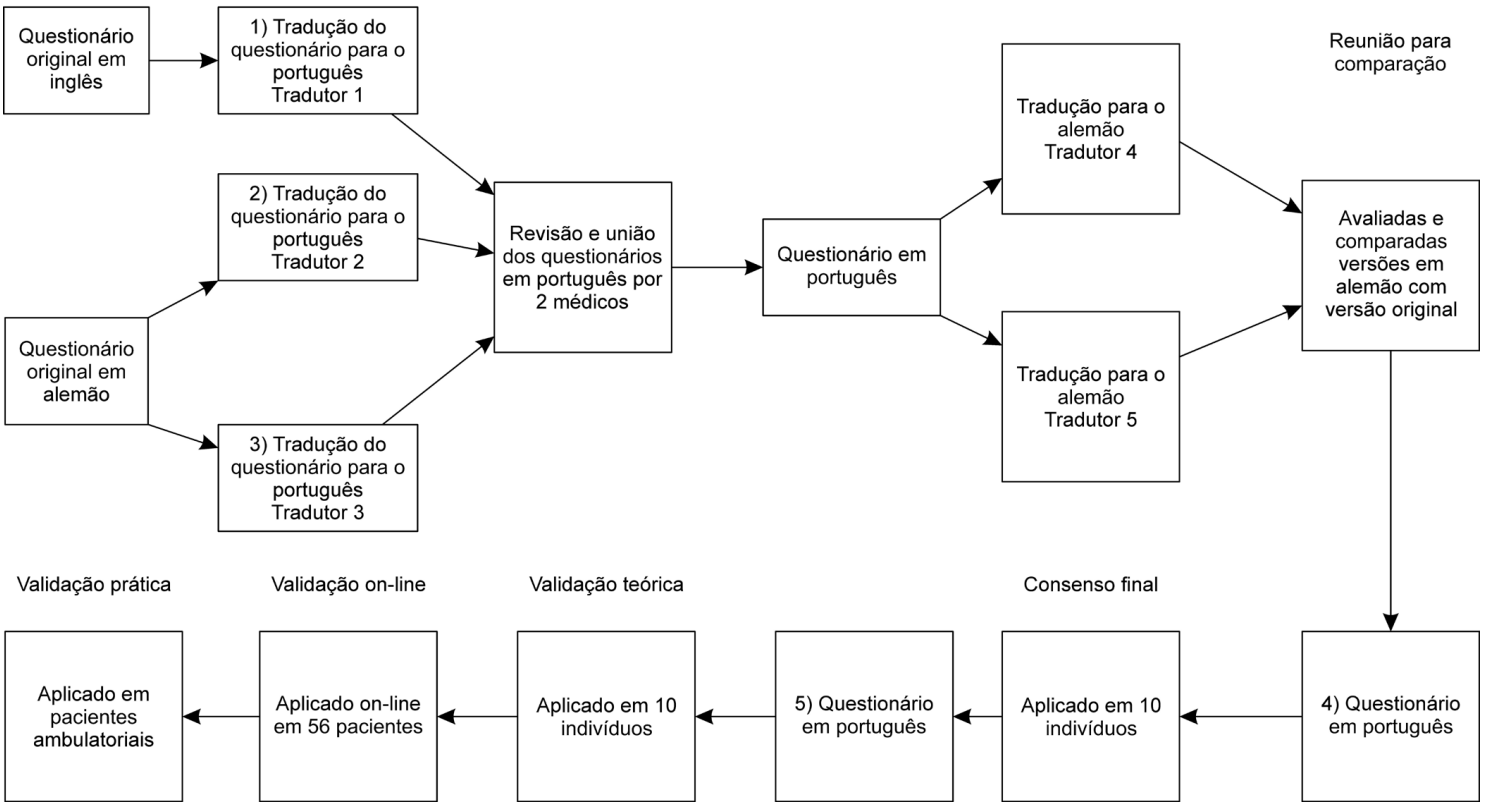

Figura 1. Fluxo de tradução, adaptação e validação.

Tabela 1. Versão final do Questionário de Avaliação Sintomática de Lipedema.

Questionário de Avaliação Sintomática do Lipedema (QuASiL)

Nome:

Data:

Gradue seus sintomas de 0 (não) a 10 (muito). Se o critério for variável, selecione a intensidade máxima que você sente.

As áreas afetadas são dolorosas? (0 não / 10 muito)

As áreas afetadas são sensíveis ao toque ou à pressão? (0 não / 10 muito)

Você tende a ter manchas roxas facilmente e frequentes nas pernas?

(Hematomas, equimoses) (0 não / 10 muito)

Você sente "pressão" ou "tensão" nas pernas? (0 não / 10 muito)

Sente as pernas "quentes" ou sensação de "queimação"?

(0 não / 10 muito)

Sente suas pernas frias? (0 não / 10 muito)

Tem câimbras musculares? (0 não / 10 muito frequente)

Sente peso nas pernas? ( 0 não / 10 muito)

Sente cansaço nas pernas? (0 não / 10 muito)

Sente inchaço nas pernas? (0 não / 10 muito)

Tem "irritações" na pele? ( 0 não / 10 muita)

Sente coceira? (0 não / 10 muita)

Tem dificuldade para caminhar? Alguma limitação de movimento?

(0 não / 10 gravemente)

Como a condição afeta sua qualidade de vida? (0 nada / 10 gravemente)

Está satisfeita com a aparência das pernas?

(Atenção: 0 muito satisfeita / 10 insatisfeita) 
local de atendimento, sendo sua equivalência cultural testada novamente até que todos os itens fossem compreendidos por $90 \%$ dos entrevistados.

A versão 5 do questionário foi convertida para versão digital on-line com software seguro e apropriado para desenvolvimento e análise de questionários (SurveyMonkey, San Mateo, California) e foi aplicada em 56 voluntárias portadoras ou não de lipedema diagnosticado.

\section{Aplicação dos questionários}

Os questionários foram aplicados individualmente, de junho a setembro de 2019, sendo os pacientes abordados previamente a consulta médica, explicados os objetivos e o conteúdo do questionário. Quando a pessoa preenchia os critérios de inclusão do estudo e tinha disponibilidade de tempo para responder imediatamente aos questionários, foi pedido o consentimento e colaboração para participar da pesquisa.

A técnica de amostragem foi não probabilística por conveniência, sendo que os sujeitos foram selecionados em ambulatório de cirurgia vascular. Os pacientes foram, então, submetidos a anamnese e exame físico não relacionados à pesquisa e exame de bioimpedância, e foram seguidos os critérios de inclusão e exclusão.

Foram incluídas mulheres, com idade maior que 18 anos, atendidas por qualquer queixa. Foram excluídos indivíduos do sexo masculino e aqueles que não assinaram termo de consentimento, que tinham condições arteriais ou venosas graves associadas e que não falassem ou compreendessem a língua portuguesa.

$\mathrm{Na}$ fase de validação on-line do questionário, voluntárias de grupo específico de portadoras de lipedema se propuseram a responder ao questionário de forma informatizada, em março de 2020, sem auxílio externo e preenchendo todos os dados de forma livre.

O estudo teve como objetivos primários a tradução e a adaptação cultural do questionário. Secundariamente, foi avaliada a correlação do escore de sintomas com variáveis indiretas da bioimpedância segmentar.

\section{Bioimpedância}

A avaliação da composição corporal segmentar foi realizada com recurso de uma balança de bioimpedância digital multiespectral, na qual a resistência e a reactância corporal foram medidas (Tanita, BC-601, Illinois, USA). As medidas fornecidas pela bioimpedância [altura, peso, índice de massa corporal (IMC)] foram automaticamente copiadas em formulário próprio e outras variáveis foram inseridas em software criado especialmente para esse propósito, onde foi calculada medida de volume de membro inferior direito (MID) e esquerdo (MIE), individualmente, considerando a densidade gordura $0,9196 \mathrm{~g} / \mathrm{mL}$ e músculo, $1,06 \mathrm{~g} / \mathrm{mL}^{15-17}$.

\section{Análise estatística}

Após verificação manual da consistência dos dados, foi feita a análise estatística. $\mathrm{O}$ método estatístico utilizado foi descritivo e de frequências. A correlação entre variáveis do questionário foi avaliada por correlação de Spearman e teste de Shapiro-Wilk. A relação entre o volume do membro e a intensidade dos sintomas no questionário foi avaliada pelo coeficiente de correlação de Pearson. A análise estatística foi realizada com auxílio do teste $t$ de Student, teste de uniformidade de Kolmogorov-Smirnov e teste de Mann-Whitney. Assumimos, para as correlações, um nível de significância estatística de 0,05\%. Os softwares utilizados para análise dos dados foram o Excel (Microsoft, Redmond, Washington, EUA) e Wizard 1.9.40 (Evan Miller, Chicago, IL, EUA).

\section{RESULTADOS}

Vinte indivíduos participaram da fase de avaliação da adaptação cultural. Nenhuma questão foi considerada como não aplicável. As questões foram compreendidas por pelo menos de $90 \%$ dos entrevistados e elas foram modificadas e reaplicadas até que todos os itens apresentassem um nível de compreensão acima de $90 \%$. A versão 5 final, aplicada on-line na população voluntária (Tabela 2), mostrou compreensão total de $96,4 \%$ da população estudada, com tempo médio de resposta de 4 minutos. Houve correlação moderada negativa entre a pergunta "Sente peso nas pernas?" e o grau de escolaridade (Spearman $\rho-0,316, p=0,018$ ), correlação fraca negativa entre a pergunta "Sente cansaço nas pernas?" e o nível de escolaridade (Spearman $\rho-0,292, p=0,029)$ e correlação moderada negativa entre a pergunta "Está satisfeita com a aparência das pernas?" e o nível de escolaridade (Spearman $\rho-0,309, p=0,02)$. A somatória dos resultados não foi normal e uniformemente distribuída (Shapiro-Wilk $\mathrm{z}=2,688, \mathrm{p}=0,004 ;$ Kolmogorov-Smirnov $\mathrm{D}=0,35$, $\mathrm{p}<0,001)$, não apresenta correlação com peso, IMC ou mesmo grau de escolaridade, mas apresenta correlação fraca negativa com a idade (Pearson $\mathrm{r}=-0,280$, $\mathrm{p}=0,037)$. Foi acrescentada escala colorida para facilitar a compreensão de intensidade.

A amostra foi constituída de 154 pacientes, que foram abordadas em ambulatório de cirurgia vascular (Tabela 3), independente do diagnóstico, realizaram bioimpedância com avaliação segmentar corporal e responderam ao questionário de avaliação sintomática de lipedema. 
Tabela 2. Características da população estudada na validação on-line da versão 5.

\begin{tabular}{|c|c|}
\hline Característica do paciente & Média (mínimo-máximo ou IC) \\
\hline Voluntárias & 56 \\
\hline Idade & $38,69(22-67)$ \\
\hline Peso & $79,46(50-125)$ \\
\hline Altura & $1,62(1,5-1,76)$ \\
\hline IMC & $30,10(20,81-45,91)$ \\
\hline Diagnóstico de lipedema prévio & $71,4 \%(I C 95 \% 58,5-81,6)$ \\
\hline Diagnóstico de varizes prévio & $73,2 \%($ IC95\% 60,4-83) \\
\hline \multicolumn{2}{|l|}{ Grau de escolaridade } \\
\hline Ensino superior completo & $35,7 \%$ \\
\hline Pós-graduação & $35,7 \%$ \\
\hline Ensino médio completo & $12,5 \%$ \\
\hline Ensino superior incompleto & $12,5 \%$ \\
\hline Ensino técnico & $3,6 \%$ \\
\hline Compreenderam integralmente o questionário & $96,4 \%($ IC95\% 87,9-99) \\
\hline Sugestões de mudança & Nenhuma \\
\hline \multicolumn{2}{|l|}{ Demografia (estado) } \\
\hline São Paulo & $60 \%$ \\
\hline Paraná & $14,5 \%$ \\
\hline Minas Gerais & $7,3 \%$ \\
\hline Rio de Janeiro & $5,5 \%$ \\
\hline Mato Grosso do Sul & $3,6 \%$ \\
\hline Rio Grande do Sul & $3,6 \%$ \\
\hline Santa Catarina & $3,6 \%$ \\
\hline Rio Grande do Norte & $1,8 \%$ \\
\hline
\end{tabular}

IC = intervalo de confiança; IMC = índice de massa corporal.

Tabela 3. Características da população estudada na validação prática.

\begin{tabular}{cc}
\hline Características do paciente & Média (mínimo-máximo) \\
\hline Pacientes & 154 \\
Idade & 43,74 anos $(19-79)$ \\
Altura & $163,86 \mathrm{~cm}(139-183)$ \\
Peso & $79,612 \mathrm{~kg}(52,1-130,3)$ \\
IMC & $29,657 \mathrm{~kg} / \mathrm{cm}^{2}(20,6-45,6)$ \\
Volume MID & $13.627,22 \mathrm{~cm}^{3}(9.051,57-24.819,61)$ \\
Volume MIE & $13.448,379 \mathrm{~cm}^{3}(8.885,915-24.757,54)$ \\
Escore sintomas & $79,091(29-148)$ \\
\hline
\end{tabular}

IMC: Índice de Massa corpórea; MID: Membro inferior direito; MIE: membro inferior esquerdo.

$\mathrm{O}$ volume dos membros direitos e esquerdo possuem médias e medianas semelhantes (teste $t$, $\mathrm{p}=0,627$; teste de Mann-Whitney, $\mathrm{p}=0,543$ ) e ambas variáveis, analisadas individualmente pelo teste de Kolmogorov-Smirnov, não estão uniformemente distribuídas, $\mathrm{p}<0,001$ (Figura 2).

$\mathrm{O}$ volume do MID possui correlação fraca positiva com a intensidade dos sintomas avaliado pelo questionário traduzido (correlação de Pearson $r=0,186, p=0,034$ ) e o volume do MIE também possui correlação fraca positiva com a intensidade dos sintomas (correlação de Pearson $r=0,183, p=0,037$ ) (Figura 3).

\section{DISCUSSÃO}

Existe muita confusão e subdiagnóstico do lipedema devido à falta de um teste de laboratório ou um teste genético definitivo e fácil, associada à falta de familiaridade médica com os critérios de diagnóstico para o lipedema ${ }^{18}$. O lipedema é mascarado por outras condições e comorbidades, como linfedema, desproporção fisiológica do formato do corpo, lipohipertrofia, obesidade ginecoide, que podem contribuir para essa confusão e levar a um diagnóstico errado ou a um subdiagnóstico ${ }^{19}$. O diagnóstico 


\section{Distribuição do volume do membro inferior direito}

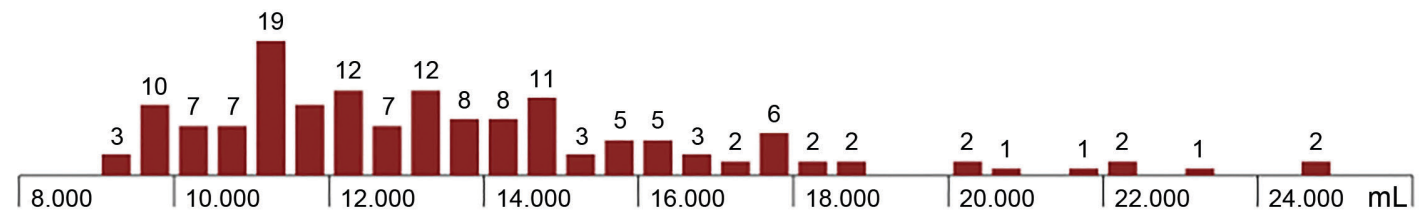

Média estimada $=13.627,222 \pm 515,781 \mathrm{~mL}$

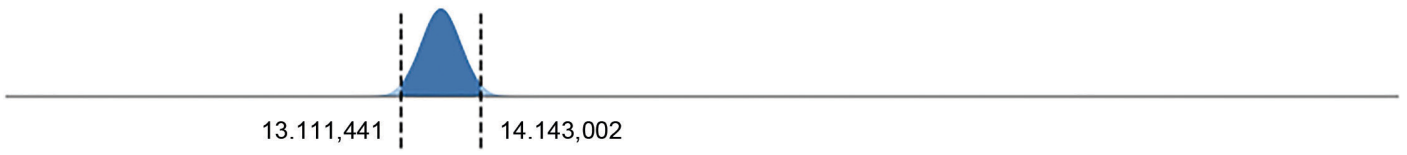

\section{Distribuição do volume do membro inferior esquerdo}

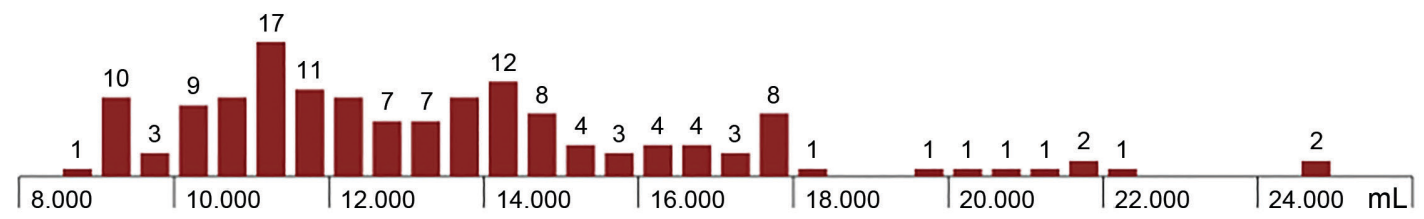

Média estimada $=13.448,379 \pm 512,286 \mathrm{~mL}$

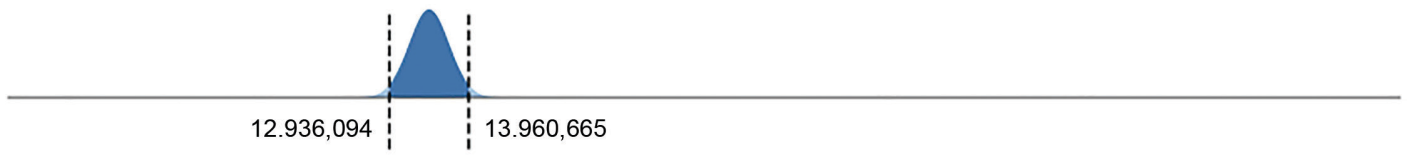

Figura 2. Distribuição do volume $(\mathrm{mL})$ dos membros e médias estimadas.
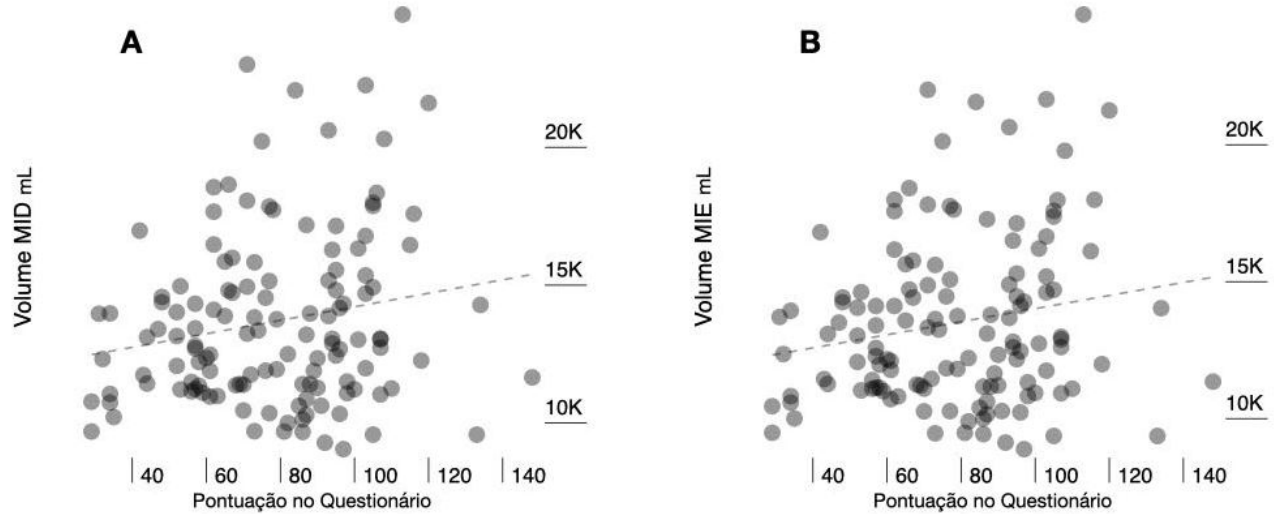

Intensidade dos Sintomas no Questionário de Avaliação

Figura 3. Intensidade dos sintomas no questionário de avaliação (somatória de pontos) correlacionada com o volume ( $\mathrm{mL}$ ) do membro inferior direito (MID) e membro inferior esquerdo (MIE).

errado em pacientes com lipedema é preocupante, pois atrasa o tratamento da doença e permite a sua progressão ${ }^{18}$. Até $50 \%$ dos pacientes com lipedema também estão em sobrepeso ou são obesos, sendo que a associação de comorbidades dificulta o diagnóstico, mas não impede que ele seja feito, pois há distinções importantes entre os cuidados para os pacientes com obesidade comum e lipedema. ${ }^{19}$ 
Sintomas inflamatórios do lipedema determinam a qualidade de vida do paciente ${ }^{20}$, e o paciente pode apresentar períodos de melhora e piora durante a vida. Atualmente não há possibilidade de acompanhar a evolução sintomática, melhora ou piora, a partir de exames laboratoriais ou de imagem no lipedema. Portanto, o questionário traduzido é uma ferramenta útil para avaliar o grau de comprometimento sintomático que o paciente se encontra e a evolução posterior, comparando-a consigo mesma.

Medir a influência na qualidade de vida da doença lipedema ainda é tarefa difícil, pois os sintomas e queixas são subjetivos e confundem-se com outras doenças muito frequentes no consultório vascular, como a insuficiência venosa crônica, obesidade e linfedema. Apesar de o assunto ter recebido atenção nos últimos anos e de já existirem vários instrumentos genéricos e específicos para realizar avaliação da qualidade de vida, tais instrumentos, na sua grande maioria, avaliam apenas os diagnósticos diferenciais, como insuficiência venosa, obesidade e linfedema. Por isso, é importante desenvolver e validar instrumentos que possam avaliar o impacto do lipedema na qualidade de vida e, se possível, auxiliar no diagnóstico definitivo.

Após a sequência de tradução, revisão, retrotradução e aplicação em população distinta do questionário, a versão final mostrou-se altamente compreensível pela população estudada $(96,4 \%)$, com correlação fraca e moderada com nível de escolaridade em apenas 3 perguntas. Correlação não implica causalidade ${ }^{21}$. Devemos ressaltar que a pergunta "Está satisfeita com a aparência das pernas?" possui escala inversa para facilidade de compreensão e padronização do questionário, ou seja, o incômodo com a aparência das pernas é maior com menor escolaridade. Não houve palavras não compreendidas. A correlação inversa da somatória dos sintomas com a idade pode sugerir melhora dos sintomas ou mesmo aceitação dos sintomas pela paciente.

A bioimpedância segmentar aplicada é um método de fácil emprego e de alta reprodutibilidade, embora fatores como posição do indivíduo, posição das manoplas, temperatura ambiente, nível de hidratação e atividade física possam afetar essa medida ${ }^{22}$. Embora o presente trabalho tenha evidenciado correlação entre a intensidade dos sintomas, avaliada pelo questionário traduzido, com o volume dos membros inferiores, que pode sugerir utilidade interindividual, a ferramenta foi desenvolvida para comparação sintomática intraindividual de melhora ou piora dos sintomas.

\section{CONCLUSÃO}

A versão do questionário visual analógico de sintomas do lipedema traduzida e adaptada culturalmente para o português do Brasil é um instrumento prático, de fácil e rápida aplicação, que pode ser utilizado na nossa população para quantificação de dados subjetivos referentes ao lipedema. Ainda são necessários estudos adicionais para avaliar a sensibilidade do instrumento para o auxílio diagnóstico do lipedema e correlação com outros aspectos do lipedema.

\section{REFERÊNCIAS}

1. Allen EV, Hines EA, Hines EA. Lipedema of the legs: a syndrome characterized by fat legs and orthostatic edema. Proc Staff Meet Mayo Clin. 1940;15:184-7.

2. Wold LE, Hines EA Jr, Allen EV. Lipedema of the legs: a syndrome characterized by fat legs and edema. Ann Intern Med. 1951;34(5):124350. http://dx.doi.org/10.7326/0003-4819-34-5-1243. PMid:14830102.

3. Amato ACM. Is lipedema a unique entity? EC Clin Med Cases Reports. 2020;2:1-7.

4. Fife CE, Maus EA, Carter MJ. Lipedema: a frequently misdiagnosed and misunderstood fatty deposition syndrome. Adv Skin Wound Care. 2010;23(2):81-92, quiz 93-4. http://dx.doi.org/10.1097/01. ASW.0000363503.92360.91. PMid:20087075.

5. Beninson J, Edelglass JW. Lipedema - the non-lymphatic masquerader. Angiology. 1984;35(8):506-10. http://dx.doi. org/10.1177/000331978403500806. PMid:6476475.

6. Reich-Schupke S, Altmeyer P, Stücker $M$. Thick legs - not always lipedema.J Dtsch Dermatol Ges. 2013;11(3):225-33. PMid:23231593.

7. Forner-Cordero I, Szolnoky G, Forner-Cordero A, Kemény L. Lipedema: an overview of its clinical manifestations, diagnosis and treatment of the disproportional fatty deposition syndrome - systematic review. Clin Obes. 2012;2(3-4):86-95. http://dx.doi. org/10.1111/j.1758-8111.2012.00045.x. PMid:25586162.

8. Okhovat J-P, Alavi A. Lipedema: a review of the literature. Int J Low Extrem Wounds. 2015;14(3):262-7. http://dx.doi. org/10.1177/1534734614554284. PMid:25326446.

9. Goodliffe JM, Ormerod JOM, Beale A, Ramcharitar S. An under-diagnosed cause of leg swelling. BMJ Case Rep. 2013;2013:bcr2013009538. http://dx.doi.org/10.1136/bcr-2013-009538. PMid:23709549.

10. Rapprich S, Dingler A, Podda M. Liposuktion ist eine wirksame Therapie beim Lipödem - Ergebnisse einer Untersuchung mit 25 Patientinnen.JDDG - J Ger Soc Dermatology. 2011;9:33-41. https:// doi.org/10.1111/j.1610-0387.2010.07504_suppl.x.

11. Rapprich S, Dingler A, Podda $M$. Liposuction is an effective treatment for lipedema-results of a study with 25 patients. J Dtsch Dermatol Ges. 2011;9(1):33-40. http://dx.doi.org/10.1111/j.16100387.2010.07504.x. PMid:21166777.

12. Augustin M, Bross F, Földi E, Vanscheidt W, Zschocke I. Development, validation and clinical use of the FLQA-I, a disease-specific quality of life questionnaire for patients with lymphedema. Vasa. 2005;34(1):31-5. http://dx.doi.org/10.1024/0301-1526.34.1.31. PMid:15786935

13. Beaton DE, Bombardier C, Guillemin F, Ferraz MB. Guidelines for the process of cross-cultural adaptation of self-report measures. Spine. 2000;25(24):3186-91. http://dx.doi.org/10.1097/00007632200012150-00014. PMid:11124735.

14. Guillemin F, Bombardier C, Beaton D. Cross-cultural adaptation of health-related quality of life measures: Literature review and proposed guidelines. J Clin Epidemiol. 1993;46(12):1417-32. http:// dx.doi.org/10.1016/0895-4356(93)90142-N. PMid:8263569.

15. Urbanchek MG, Picken EB, Kalliainen LK, Kuzon WM Jr. Specific force deficit in skeletal muscles of old rats is partially explained 
by the existence of denervated muscle fibers. J Gerontol A Biol Sci Med Sci. 2001;56(5):B191-7. http://dx.doi.org/10.1093/ gerona/56.5.B191. PMid:11320099.

16. Farvid MS, Ng TWK, Chan DC, Barrett PH, Watts GF. Association of adiponectin and resistin with adipose tissue compartments, insulin resistance and dyslipidaemia. Diabetes Obes Metab. 2005;7(4):406-13. http://dx.doi.org/10.1111/j.1463-1326.2004.00410.x. PMid:15955127.

17. Cavezzi A, Schingale F, Elio C. Limb volume measurement: From the past methods to optoelectronic technologies, bioimpedance analysis and laser-based devices. Int Angiol. 2010;29(5):392-4. PMid:20924339.

18. Dayan E, Kim JN, Smith ML, et al. Lipedema - the disease they call FAT: an overview for clinicians. USA: Lipedema Simplified Publications; 2017.

19. Child AH, Gordon KD, Sharpe P, et al. Lipedema: an inherited condition. Am J Med Genet A. 2010;152A(4):970-6. http://dx.doi. org/10.1002/ajmg.a.33313. PMid:20358611.

20. Neves AAG, Oliveira AGNM, Beck RT, Santos RV, Moreira FCP, Amato ACM. Endovascular treatment of thoracic aortic pseudoaneurysm with aortobronchial fistula in the late postoperative period of surgical correction of the aortic coarctation. J Vasc Bras. 2011;10(1):64-7. http://dx.doi.org/10.1590/S1677-54492011000100012.

21. Akoglu H. User's guide to correlation coefficients. Turk J Emerg Med. 2018;18(3):91-3. http://dx.doi.org/10.1016/j.tjem.2018.08.001. PMid:30191186.

22. Deurenberg P, Van Der Kooij K, Evers P, Hulshof T. Assessment of body composition by bioelectrical impedance in a population aged greater than 60 y. Am J Clin Nutr. 1990;51(1):3-6. http:// dx.doi.org/10.1093/ajcn/51.1.3. PMid:2296928.
Correspondência Alexandre Campos Moraes Amato Av. Brasil, 2283 - Jardim América CEP 01431-001 - São Paulo (SP), Brasil E-mail: dr.alexandre@amato.com.br

Informações sobre os autores ACMA - Doutor em Ciências, Universidade de São Paulo (USP); Professor, Disciplina de Cirurgia Vascular, Universidade de Santo Amaro (UNISA); Titular, Sociedade Brasileira de Angiologia e Cirurgia Vascular; Especialista em Cirurgia Vascular e Endovascular, Sociedade Brasileira de Angiologia e Cirurgia Vascular e especialista em EcoDoppler Vascular, Colégio Brasileiro de Radiologia.

FCMA - Mestre em Ciências, Universidade Federal de São Paulo

(UNIFESP) e Cirurgião Plástico.

DAB - Cirurgião Vascular Chefe, Departamento de Cirurgia Vascular e Endovascular, Medical Valens Center.

RVS - Cirurgião Vascular e Mestre em Ciências, Universidade de São Paulo (USP).

Contribuições dos autores Concepção e desenho do estudo: ACMA, DAB Análise e interpretação dos dados: $A C M A, D A B$ Coleta de dados: ACMA, DAB, FCMA Redação do artigo: ACMA, DAB Revisão crítica do texto: ACMA, DAB, RVS, FCMA Aprovação final do artigo*: ACMA, DAB, RVS, FCMA Análise estatística: ACMA Responsabilidade geral pelo estudo: ACMA, DAB, RVS, FCMA

*Todos os autores leram e aprovaram a versão final submetida ao J Vasc Bras. 